\title{
The community capacity building for sustainable dengue problem solution (CCB-SDPS) model: The results from studies in community, southern region, Thailand ${ }^{*}$
}

\section{Charuai Suwanbamrung}

School of Nursing, Walailak University, Nakhorn Si Thammarat Province, Thailand; Scharuai@wu.ac.th

Received 6 January 2012; revised 8 February 2012; accepted 11 March 2012

\section{ABSTRACT}

This paper provides an approach for sustainable dengue problem solution by the community capacity building model and the results of two studies from implementation the model in Southern region, Thailand. The conceptual framework of the CCB-SDPS model consisted of three dimensions; 1) community-based (leader and nonleader in community), 2) community capacity building process, and 3) sustainable dengue problem solution (outcomes). The outcomes of studies focused on the capacity level by the DCCAT (14 domains of leader group, and 11 domains of non-leader group), dengue entomology (Larval) index, and epidemiological (morbidity and mortality) rate. The two studies were pilot study in high risk dengue areas. These studies were application of CCB-SDPS model. First study was the process of using the tool for testing the DCCAT in two communities. The results showed only three steps of using assessment tool; community preparation, assessment, and community hearing. The second study was the fully testing model in three communities. Participatory action research and mix methods; quantitative and qualitative collecting data were preparation community-based, building capacity process (assessment, plan, implement, and reassessment), and outcomes monitoring. The results of second study showed three models of the CCB-SDPS. The two studies confirmed the sustainable in after intervention of community capacity building such as increased capacity level of leader and non-leader group, deceased entomological indices ( $\mathrm{HI}, \mathrm{BI}$, and $\mathrm{Cl}$ ), and non

\footnotetext{
*Conflict of Interest: The author(s) declare that they have no competing
} interests. presentation of morbidity and mortality rate in during study period. These results showed the model conducing on contexts of each community such the three dimensions of the conceptual framework of the model, community-based, building capacity process, and measurement of outcomes of sustainable dengue problem solution. These experiences in Southern Thailand demonstrated the main keys to the approach of CCB-SDPS were in the use of strategies planning to initiate community capacity building towards sustained dengue problem solution.

Keywords: Community Capacity Building; Sustainable Dengue Problem Solution; Dengue Community-Based; DCCAT; CCB-SDPS

\section{INTRODUCTION}

Dengue is a public problem requiring the community’s involvement in its solution. Sustainability is a major issue of community-based dengue prevention and control, defined according to the characteristics of each specific setting [1,2]. In this study, sustainability is identified as a necessary part of successful community-based dengue programs. Sustainability must be evaluated by ongoing activities of leaders and non-leaders in the community, as well as at the outcome of a program [3-5].

To achieve sustainability, community capacity building is one of the instrumental factors; it contributes to the process of enhancing a community's abilities to define, evaluate, analyze and act on the dengue problem [6-9]. Capacity building is a "concept both of the means and the end" [10] and, as a dynamic process, it must be part of the strategy for sustainability of the intervention from the beginning to the end [11]. With a means or standard for its measurement, community health interventions would result in increased sustainability and capacity for 
future problem solving. Thus, the identification and assessment of community capacity, is important to those striving to develop healthy communities. Unfortunately, there are currently few tools to assess the capacity of the community for sustainable community-based dengue prevention and control and its qualitative assessment [11].

In Thailand, the national dengue control and prevention program has endorsed community-based control programs by encouraging residents to take responsibility for control activities in households. However, current dengue prevention and control activities have not had much impact. Southern Thailand is at high risk of dengue transmission because there are several factors which favor dengue incidence: more rainy days, greater total rainfall, higher average relative humidity, and warmer temperatures [12]. The high incidence of dengue in the Southern Thailand community requires a strengthening of community action by building the capacity of all affected groups. People need a better understanding of measures for the prevention and control of the disease.

To conduct the appropriate of community capacity building for sustainable community-based dengue prevention and control, the leaders and non-leaders in the community need to identify appropriate tools and to assess community capacity in various domains. Although a new tool to assess the community capacity for sustainable community-based dengue prevention and control was very much needed, such a tool was not available. Thus, this article would like to present the community capacity building model and results of the two implementation studies in high risk dengue community.

\section{THE COMMUNITY CAPACITY BUILDING MODEL}

The model of community capacity for a sustainable solution to the problem of dengue in this study consists of three dimensions: community-based dengue prevention and control (leaders and non-leaders), a community capacity building process, and sustainable communitybased dengue prevention and control.

\subsection{Community-Based Dengue Prevention and Control}

A community-based dengue prevention and control process enables key stakeholders in the community to actively prevent and control their dengue problem. The strategies of dengue prevention and control at the sub-district level focuses on vector control and transmission of infections to humans, based on the community as the setting, target, agent and resources for dengue activities $[4,13]$.

In this study, community-based dengue prevention and control was analyzed in three communities with dengue focusing on two groups for dengue prevention and control: the first group was the leader group who assumed the role as the "capacity building activities group" and consisted of representatives of dengue health promoters, local authority/organization networks, schools, temples, and village health volunteers. The second group was the non-leader group whose role was as the "sustainable prevention and control activities group" and consisted of community members (see Figure 1 for participants on community-based).

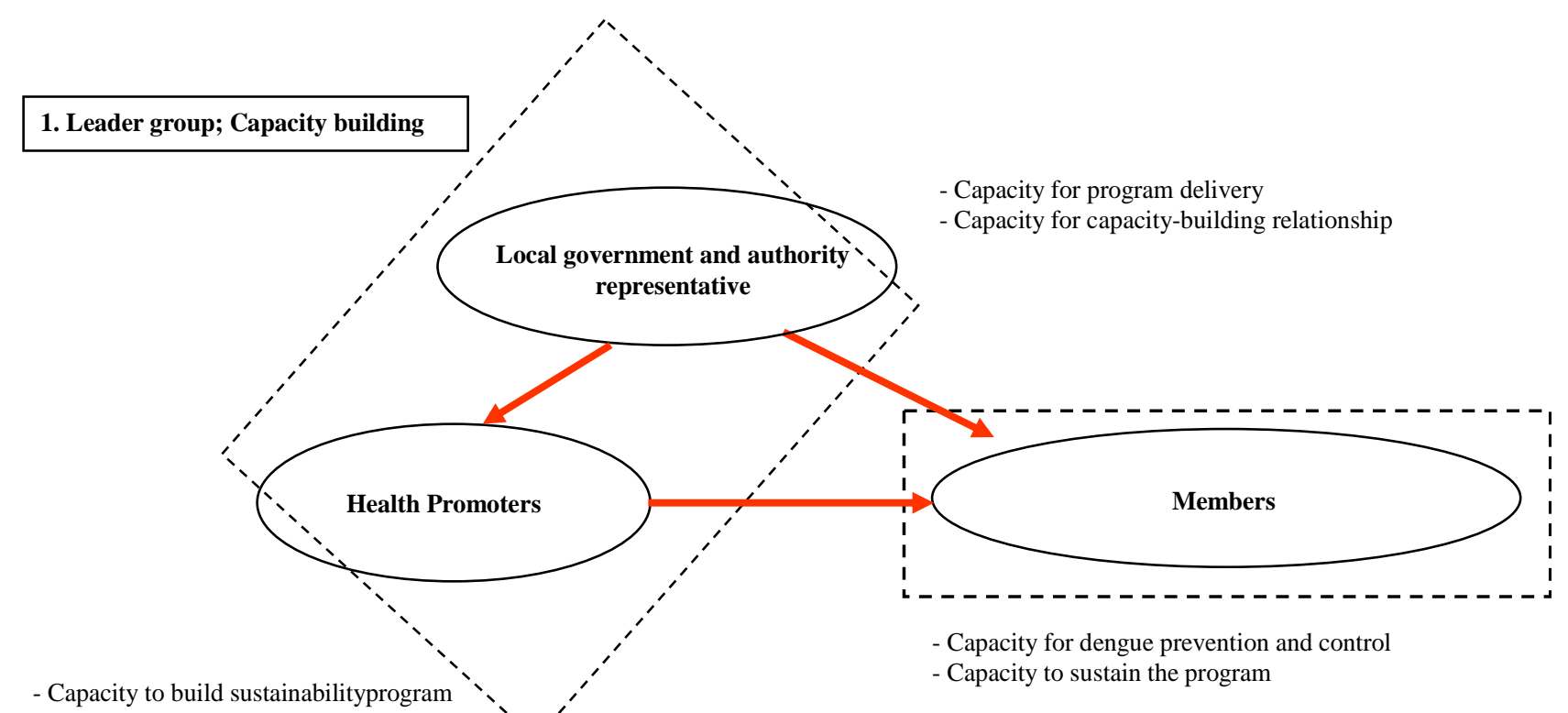

- Capacity building relationship

2. Non-leader group; Sustainable prevention and control

Figure 1. Community-based relationship for building community capacity to overcome the dengue problem. 


\subsection{Community Capacity Building Process}

Community capacity building is a process which demonstrates an increase in the various domains of community capacity in a community-based dengue prevention and control program. The processes of community capacity building involves the following steps: 1) preparation (Develop the operational domain and prepare to assess the community capacity); 2) assessment of community capacity 3) development of a strategic plan and implementation and 4) follow-up or reassessment [13-17]. In this study, dengue community capacity building is defined as a process of building community capacity for dengue prevention and control in community involving 4 steps such as 1) community preparation, 2) assessment, 3) plan and implement and 4) re-assessment.

In the process of the community capacity building for sustainable dengue prevention and control was the community capacity domains of the leader group (14 domains) and non-leader group (11 domains) $[18,19]$. There were three domains of the leader group more than the non-leader group-Leaders group networking domain, Leaders group and community networking domain, and Community participation domain as following:

\subsubsection{Critical Situation Management Domain}

The critical situation management is a distinctive capacity domain because it is the first domain of both groups. The critical situation management domain of the leader group includes nine capacities whereas this domain of the non-leader group consists of 13 sub-capacities. There are five capacities which overlap in both groups. The relevant capacities of critical management domain focused on key dengue stakeholders and their activities in quickly prevention and control dengue problem.

\subsubsection{Personal Leadership Domain}

The personal leadership is the second domain of both groups. The domain in the leader group includes 12 sub-capacities and the non-leader group consists of eight sub-capacities. An examination of content related to this domain focuses on individual perception of their activities to prevent and control dengue disease. For the leader' group, there are five activities focusing on capacities to enhance other dengue stakeholders for dengue prevention and control.

\subsubsection{Health Care Provider Capacity Domain}

This was the third domain of the leader group (8 sub-capacities) and the fifth domain of the non-leader group (6 sub-capacities). An examination of the activities content reveals that these sub-capacities focused on dengue prevention and control activities of health care workers and village health volunteers. Five sub-capacities overlapped in both groups. These capacities indicated the important capacity of village health volunteers for dengue prevention and control because village health volunteers are key stakeholders of the health care service in the community in Thailand.

\subsubsection{Needs Assessment Domain}

This domain is the fourth domain of the leader group (8 sub-capacities) and the eleventh of the non-leader group (5 sub-capacities). There were four sub-capacities which overlapped in both groups. An examination of the sub-capacities showed that these sub-capacities focused on community members' needs related to the dengue problem and its solution. These sub-capacities indicated the importance of the local administrative organization as a centre of the dengue solution provider.

\subsubsection{Senses of Community Domain}

This domain is the fifth domain of the leader group (11 sub-capacities) and the sixth domain for the nonleader group (8 sub-capacities). There are seven subcapacities which overlapped both groups. An examination of capacities content indicated that these capacities focused on perception of the dengue problem and the solution in the community.

\subsubsection{Leaders Group Networking Domain}

This domain is the sixth domain for only leader group included 11 sub-capacities An examination of the capacity content of these sub-capacities indicated a focus on the individual dengue network of the leader group members with representatives of other stakeholders.

\subsubsection{Communication of Dengue Information Domain}

This domain is the seventh capacity domain of leader group (10 sub-capacities) and the seventh domain of the non-leader group (7 sub-capacities). An examination of the sub-capacities content indicated that these sub-capacities focused on channels and resources of receiving dengue information. Four sub-capacities overlapped in both groups focusing on familiar channels in sub-districts of Southern Thailand.

\subsubsection{Community Leadership Domain}

This domain is the eighth capacity domain of the leader group (8 sub-capacities) and the fourth domain of the non-leader group (8 sub-capacities). Almost all activities (7 of 8 sub-capacities) in the non-leader and leader overlapped in both groups. An examination of the capacities content related to these sub-capacities focused on the community members' perception of dengue prevention and control as their responsibility. The overall 
group perception in the community of community leadership is a person who shows strength, consults, manages, accepts clear responsibility, listens, and focuses attention on dengue prevention and control.

\subsubsection{Religious Capacity Domain}

This domain is the ninth capacity domain of the leader group (9 sub-capacities) and the third capacity domain of the non-leader group (10 sub-capacities). There are nine sub-capacities which overlap in both groups. An examination of activities content related to these sub-capacities focused on the capacity of imams and monks to undertake activities of dengue prevention and control.

\subsubsection{Leaders Group and Community Networking Domain}

The tenth domain of only the leader group contained of seven sub-capacities. An examination of the capacity content focused on dengue prevention and control by networking between community members and leaders. Leader and non-leader groups both participate for dengue prevention and control activities.

\subsubsection{Resource Mobilization Domain}

This is the eleventh capacity domain of the leader (4 sub-capacities) and the tenth domain of the non-leader (5 sub-capacities). Four sub-capacities overlapped in both sub-tools. An examination of the sub-capacities content revealed that these focused on the ability of the community's members to mobilize resources for dengue prevention and control.

\subsubsection{Dengue Working Group Domain}

This domain is the twelfth capacity domain of leader (6 sub-capacities) and the ninth capacity domain of the non-leader (7 sub-capacities). There were five sub-capacities which overlapped both groups. An examination of the sub-capacities content indicated that these capacities focused on the community member group and representatives of organizations in the community as the leader group to prevent and control dengue disease. The core leader means the community group which takes the lead in capacity building for dengue prevention and control.

\subsubsection{Community Participation Domain}

The thirteenth capacity domain of only the leader group is community participation. This domain consisted of six sub-capacities. An examination of the sub-capacities content suggested that these focused on community leader's participation in dengue prevention and control. Community participation (CP) is the most important strategy in dengue management.

\subsubsection{Continuing Activities Domain}

This domain is the fourteenth capacity domain of leader (6 sub-capacities) and the eighth capacity domain of non-leader (6 sub-capacities). An examination of the capacities content indicated these capacities focused on community guidelines and policies of dengue prevention and control. Four sub-capacities overlapped in both groups.

\subsection{Sustainable Community-Base of Dengue Prevention and Control}

Community-based dengue prevention and control comprise activities through which people to control and eliminate larval breeding sources, control adult mosquitoes, apply personal protection, introduce dengue symptom detection and outbreak prevention [4]. They were measured by assessing the effective performance in specific community capacity domains, exhibiting dengue prevention and control behaviors as continuing evidence of implementing dengue strategies or activities, and the results of such 1) dengue community capacity level of the leader group (14 domains) and the non-leader group (11 domains), 2) dengue entomology index; Larval Indices such as Breteau Index (BI), House Index (HI), and Container Index (CI), and 3) dengue epidemiology index; morbidity rate and mortality rate[4,20-22]. These dimensions can be seen as Figure 2.

\section{DENGUE COMMUNITY CAPACITY ASSESSMENT TOOL (DCCAT) [23]}

The mixed methodological approach was two phases in Southern Thailand. Methods: The first phase was defining meaning and themes of dengue community capacity domains by qualitative method, 10 initial dengue community domains were identified, by means of the three experts reviews with content analysis from literature reviews, in-depth interviews with 60 leaders, and 8 focus group discussions with 60 non-leaders, in 4 sub- districts of the 4 provinces. The second phase was developing items and testing tool phase. It was divided into dengue community capacity for leaders and non-leaders. Content validity was verified by a seven-expert review panel, which arrived at a total Content Validity Index (CVI) of leaders (0.90) and non-leaders (0.91). The items were measured with a 5-point rating scale. During the pilot-testing, the Conbrach alpha reliability coefficient for the leaders was 0.98 and the non-leaders it was 0.97 . Items were revised leaders items 182 and non-leaders items 167 . The testing tools were administered to 964 leaders and 1248 non-leaders, throughout 8 sub-districts of the 8 provinces. Construct validity was analyzed by Exploratory Factor Analysis (EFA). Factor loading was 0.5 , the Eigenvalue was greater than 2, and all the domains 
together explained in leaders $57.58 \%$ and non-leaders $57.11 \%$ of the variance. The findings were Domains and Dengue Community Capacity Assessment Tool of leaders (DCCAT-L) and non-leaders (DCCAT-NL). DCCATL was 14 domains (115-item) and DCCAT-NL was 11 domains (83-item). The DCCAT-L was three more domains than the non-leaders, which included leaders group networking, leaders group and community networking, and community participation domain. The DCCAT is the new tool which consonants the previous concepts and a few study. It can be used to assess and reassess community in improving community capacity for sustainable dengue prevention and control.

As key actors in community, these two groups were the key players in conducting dengue prevention and control in community. There was an overlap of 58 items between dengue community capacity domains of nonleaders (11 domains and 83 items) and those of the leaders (14 domains and 115 items). They were as follows: critical situation management (5 items), personal leadership (4 items), religious leader capacity (9 items), community leadership (7 items), health care provider capacity (5 items), sense of community, (7items), communication of dengue information (4 items), continuing activities (4 items), dengue working group (5 items), resource mobilization (4 items), and needs assessment (4 items). All domains of DCCAT-L, DCCAT-NL and the overlapping items of both groups are shown in Table 1.

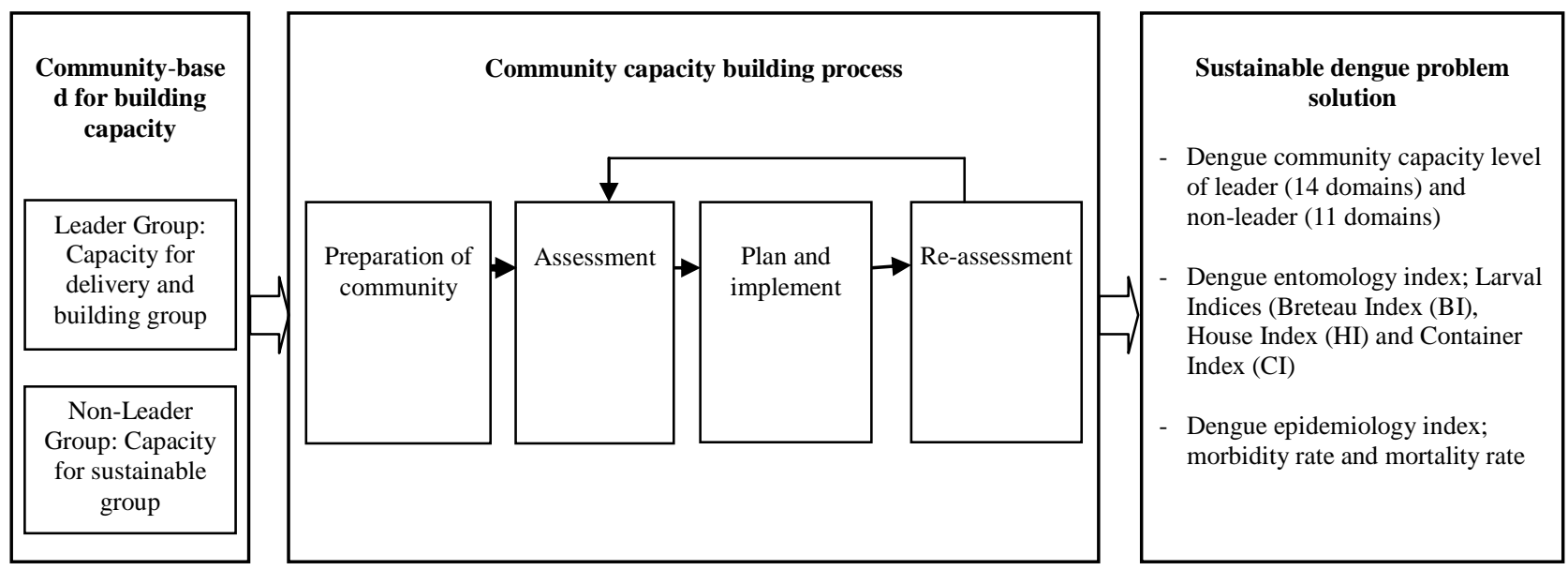

Figure 2. Community capacity building model for sustainable dengue problem solution.

Table 1. Comparing domains of DCCAT and overlapping items.

\begin{tabular}{|c|c|c|c|c|}
\hline \multicolumn{2}{|l|}{ DCCAT-L } & \multicolumn{2}{|l|}{ DCCAT-NL } & \multirow{2}{*}{$\begin{array}{c}\text { Item } \\
\text { overlap }\end{array}$} \\
\hline Domains & Item & Domains & Item & \\
\hline 1.Critical situation management (L1) & 9 & 1.Critical situation management (NL1) & 13 & 5 \\
\hline 2. Personal leadership ( $L 2)$ & 12 & 2. Personal leadership (NL2) & 8 & 4 \\
\hline 3. Religious capacity (L9) & 9 & 3. Religious capacity (NL3) & 10 & 9 \\
\hline 5. Health care provider capacity (L3) & 8 & 5. Health care provider Capacity (NL5) & 6 & 5 \\
\hline 6. Sense of community (L5) & 11 & 6. Sense of community ( $N L 6)$ & 8 & 7 \\
\hline 7. Communication of dengue information (L7) & 10 & 7. Communication of dengue information (NL7) & 7 & 4 \\
\hline 8. Continuing activities (L14) & 6 & 8. Continuing activities ( $N L 8)$ & 6 & 4 \\
\hline 9. Dengue working group (L12) & 6 & 9. Dengue working group (NL9) & 7 & 5 \\
\hline 11. Needs assessment $(L 4)$ & 8 & 11.Needs assessment (NL11) & 5 & 4 \\
\hline 12. Leaders group networking ( $L 6)$ & 11 & & & \\
\hline 13. Leaders group and community networking ( $L 10)$ & 7 & & & \\
\hline 14.Community participation (L13) & 6 & & & \\
\hline Total 14 Domains & 115 items & Total 11 Domains & 83 items & 58 items \\
\hline
\end{tabular}




\section{TWO STUDIES OF THE IMPLEMENTATION STUDY OF THE CCB-SDPS}

\subsection{First Study: Community Capacity for Sustainable Community-Based Dengue Prevention and Control: Study of a Sub-District in Southern, Thailand}

The study was studied in 2009-2010 [18]. The objectives of this study were to assess the level of community capacity for dengue prevention and control in two communities, Southern, Thailand. A cross-sectional survey was designed by community participatory approach for use with two communities which a dengue incidence rate higher than the standard over the past five years. Data gathering was conducted by the dengue leader group (DLG), which included fifteen leaders and fifteen nonleaders who were trained by the research team. The assessment tool used the DCCAT for leaders (115-item, 14-domain) and non-leaders (83-item, 11-domain).

Participants were selected by the DLG based on their communities' dengue risk: in the low-dengue incidence (LDI) community (Ban Pakpayin community), thirty-two leaders and 177 non-leaders were selected, whereas thirty-one leaders and 199 non-leaders were chosen from the high-dengue incidence (HDI) community (Ban Pakpoon community). The method of data collection was use community participatory approach. It consisted of three steps, namely, community preparation, assessment, and community hearing.

First of all, the community preparation step consisted of consulting and discussing, setting up of the Dengue Leader Group (DLG) and the dengue support team: 1) consultation and discussions were held with the formal leaders of the sub-district i.e. health care workers, local administrative organization officers, and the formal community leaders. Morbidity and mortality of dengue from secondary for the past 5 years were discussed and a consensus of solutions of the problems reached; 2) setting up the "Dengue Leader Group" (DLG) as the key group for conducting dengue prevention and control consisting of volunteers with available time for dengue activities. The DLG included individuals from the leaders and nonleaders groups; 3) the dengue support team consisted of health workers, local administrative organization officers, and religious leaders. Second, the assessment step comprised collecting data, estimating sample size, assessing data and data analysis: 1) collecting data team. The village health volunteers were given training in data gathering skills, describing the objectives of the study and the benefits of the results of the plan and of implementing dengue prevention and control; 2) estimating sample size of leaders and non-leaders. The number of participants based on the context of the community; 3) assessing data with the DCCAT. The format consisted of 4 parts: a) general characteristics; b) two dengue community capacity-assessment questionnaires, one for the leaders (consisting of 14 domains and 115 items) and the other for non-leaders (11 domains and 83 items); c) household environment observation and d) larval indices survey form; and 4) data analysis followed the assessment format. The level of dengue community capacity of leaders and non-leaders had clear cut-off points of mean scores of each domain and total score.

Third, community consensus step involved a discussion among the DLG, support team and other stakeholders. The results of data collection can be used in this step as a base for planning and setting strategies for dengue prevention and control. The study comprised only three steps, but the two additional steps, plan and implement, and reassessment, were offered by the community as the required next step for building community capacity for sustainable dengue prevention and control.

Results of the study showed only with the leaders from the LDI and the HDI communities demonstrated high levels of dengue community-capacity ( $\bar{X} \pm$ SD $=360.47$ \pm 58.82; $418.22 \pm 57.72$ ). The non-leaders in the LDI community demonstrated a moderate level of dengue community capacity competence $(\bar{X} \pm \mathrm{SD}=205.90 \pm$ 60.76) while the non-leaders in the HDI community had a high level $(\bar{X} \pm \mathrm{SD}=254.78 \pm 50.34)$. These initial levels of dengue community capacity served as a baseline for diagnosing each community. For a community that needed to improve its capacity, it was essential to conduct a pre-post intervention assessment or a serial assessment.

The study was the application of using the DCCAT for measuring the level of community capacity of both communities (leader and non-leader group). These results confirmed that the DCCAT can measure the level of dengue community capacity based on community preparation, assessment, and community consensus.

\subsection{Second Study: A Model of Community Capacity Building for Sustainable Dengue Problem Solution in Southern, Thailand}

Community capacity building is a strategy that enhancing the community's abilities (leader and non-leader) for sustainability of dengue problem solution. Objectives: To develop the model of community capacity building for dengue problem solution and compare community capacity level before and after intervention. This study was conducting on October, 2009-October, 2010 [24].

Participatory Action Research was designed for building community capacity in three communities which high 
risk dengue areas in southern province, Thailand. The process of community capacity building was conducting on 5 steps; community preparation based on leader and non-leader group; assessment dengue situation (larval indices) and community capacity level by the DCCAT; strategies plan; implement of intervention; reassessment and conclusion. Community capacity level in each community was compared assessment and reassessment by independent $t$-test and ANOVA for compare the results among three communities. Leader and non- leader group, Ban Mon, Ban Nanghraya, and Ban Kang model were the leader group (26, 24, and 28) and the non-leader group (200, 215, and 176).

The research team, support team, and the leader group used discussion techniques and carried out analysis of the dengue problem in each model over a 13 months period. The leader group in the three models planned the interventions and partial interventions for each model as well as joint interventions for all three models. Both groups volunteered to participate in the dengue capacity building process.

The three models followed the concept of the dengue community capacity building process with its four steps: preparation, assessment, planning and implementation, and re-assessment. Meetings of the leader and non-leader groups for all four steps of activities were held at least once monthly throughout the study. Planning and implement activities consensus of the community based on their particular context and the resources in their community.

Each community developed a complete action plan and implemented activities appropriate to the context of their communities.

1) Ban Mon model, it was a village at a crossroads community selected by representatives of the local administrative organization, health center, community leader, religion leader, and village health volunteers. The community consisted of 320 households, a health center, a utility building, a temple, and a community school. Most households were situated near a large road which was the crossroads of a semi-urban community and had 4 to 6 months with rainy day in a year in an area that was low with still water in several areas. Ban Mon model implemented four activities based on the specific problems and available community resources such as conducting a dengue prevention and control campaign, communication from their community leaders, obtaining community consensus for dengue prevention and control from local administrative organizations, and meeting of the dengue leaders group once mouth. The model had eight issues that showed up in the pre-test: a) poor environment; b) community needs more of everything; c) lack of capacity of health center officer; d) misconceptions in the of use of chemical insecticides; e) deficit of dengue knowledge; f) community resources management; g) low community participation; and h) lack of continuity. For example, some participants said that chemical fogging teams showed a lack of knowledge in using chemicals which were used in fogging by such statements as if there were an outbreak of dengue illness, there "needs to be chemical fogging 2 - 3 times per month" and "needs fogging frequency". After intervention, leaders and non-leaders' suggestions in Ban Mon were fewer than pre and post-intervention: a) poor environment; b) capacity of health center official; c) misconception in using chemical; d) deficit of dengue knowledge; e) community participation. For example, some participants said: "no clear dengue information provided" "should give information for every household in community and take real survey".

2) Ban Nangpraya model, it was a seaside community in Pak Na Khorn sub-district selected by representatives of all stakeholders in the community. The community was semi-urban; with households situated closely and with more than 10 houses per group. It consisted of total of 344 households, an all-purpose building, a temple, a community school, a health center, a local district organization, community leaders, and village health volunteers. Fishing and unskilled labor was the occupation of most of the community. The model was three special activities-community communication of dengue knowledge, employing red lime for use in water containers as in the community. There were many water containers per house, meeting of DLG once a month to monitor and evaluate the program. In Ban Nangpraya community, leader and non-leader groups pointed out six issues: a) poor environment because of low land and more water containers; b) community needs all stakeholders to solve the dengue problem; c) dengue information communication; d) deficit of capacity of health center officials; e) dengue knowledge of community; and f) low community participation. For example, some participant said: "there should be meeting of dengue management among people, VHVs and health center official...", "most people in community had little knowledge of dengue", "breeding sites were many...", "no clear dengue campaigns whereby people receive true information” “...no government officials who were really responsibility so people in community are not attentive for prevention and disease control" "No promotion of dengue campaign and dengue information transfer...needs VHVs help to inform about dengue prevention and control". The intervention finished the post-intervention showed decreased suggestions of only four issues: a) poor environment; b) lack of knowledge of dengue prevention and control; c) lack of capacity of health center officials; and d) low community participation. For example, some participant said: "community has more forests and canals... cannot 
cover the entire area... villagers help only their own households to cover water containers and cultivate citronella" "needs to broadcast dengue information... increased broadcasts are beneficial”.

3) Ban Kang model, it was a small village in a subdistrict near Meung district consisting of 239 households, an all-purpose building, a temple, a mosque, a community school, a health center, a local district organization, community leaders, and village health volunteers. There were two religions; Buddhism (70\%) and Muslim (30\%). Most people in the community were rubber trappers and fruit farmers with the gardens of risk as mosquito breeding place. The model carried out three activities - a mobile meeting of the DLG conducted in each area of the community, mass communication from leaders of religion and the community leaders, and door to door survey of larval index once a month by DLG. Moreover, there were the strongest of leader and non-leader participation in all activities such as environment in and out houses for elimination dengue sources. The initially identified issues of Ban Kang model were six issues such as: a) not clear who are the VHVs and health center official, b) community participation needs to be strengthened, c) lack of continuity of activities, d) unclear dengue information, e) misconceptions concerning chemical fogging, and f) unclear community consensus. At the completion of the intervention process, the community had fewer suggestions from the leader and non-leader groups. The major issues were a partially poor environment, lack of community participation, and inadequate dengue information communication.

The community capacity building model can be increasing level of community capacity. Key element of three community capacity models for sustainable of dengue problem solution were; Tha Raue model, Pak Nakorn model, and Kumpench Sou model. Significant of community capacity level in each community were different before and after intervention $(p<0.05)$. Community capacity level among three models were significant difference $(p<0.05)$. The model of CCB-SDPS was based on the context of community such as community capacity level, situation of dengue problem, resources, and participation of leader and non-leader in each community.

\section{CONCLUSIONS}

The Community Capacity Building Model for Sustainable Dengue Problem Solution (CCB-SDPS) was three dimensions: 1) community-based dimension was participation approach which consisted of leader and non-leader group in community; 2) community capacity building process dimension was four steps such as preparation community, assessment, plan and implement, and reassessment; and 3) outcomes for measure for sustainable dengue problem solution. The model can be confirmation as the strategies for sustainable prevention and control dengue in community [18,19,23-26]. Its model conducting on preparation community, assessment capacity level based on community capacity domains of leader and non-leader group [25]. The DCCAT is the validity and reliability assessment tool for assessment on before and after of building community capacity intervention [23]. The process of the community capacity building is continuing process as the model of a continuous improvement cyclical model for vector and dengue control program which proactive strategies [27]. However the implementation of model needs the participation and understands of community which showing dengue problem. The outcomes of the CCB-SDPS were measured the entomological indices as classical index in short time and mobility rate in middle time [28]. The sustainable dengue problem solution was discussion based on long time such as 3 - 5 years after finished the project and continuous monitor [29].

\section{LIMITATION}

One limitation of the article is that it was conducted only two studies. It needs more study and continuing results after end of the project. Nevertheless, the first study was confirming steps of using only the DCCAT and the second study was completed. Another limitation was the adoption of the HI, BI, and CI as they were easier to collect and to interpret than other entomological measures, and that the leader group perceived ownership of own community and provided available participation.

\section{ACKNOWLEDGEMENT}

Grateful acknowledgements are to participants (leader and nonleader group), teachers, and representative of communities surrounding school and research team. The author(s) would like to thank Walailak University, Thailand, and the School of Nursing for the support by providing available time. Special thanks to Victor Greenspoon who has edited the manuscript.

\section{REFERENCES}

[1] Swaddiwudhipong, W., et al. (1992) Effect of health education on community participation in control of dengue hemorrhagic fever in an urban area of Thailand. The Southeast Asian Journal of Tropical Medicine and Public Health, 23, 200-206.

[2] Spiegel, J., et al. (2005) Barriers and Bridges to prevention and control of dengue: The need for a social for a social-ecological approach. EcoHealth, 2, 273-290. doi:10.1007/s10393-005-8388-x

[3] McKenzie, J.F., Neiger, B.L. and Smeltzer, J.L. (2005) Planning, implementing, and evaluating health promotion 
programs. Preson Education, Inc., San Franciaco.

[4] WHO (1999) Prevention and control of dengue and dengue hemorrhagic fever: Comprehensive guidelines. WHO Regional Publication, New Delhi.

[5] Windsor, R., et al. (2003) Evaluation of health promotion, health education, and disease prevention programs. Mc Graw Hill higher Education, Boston Burr Ridge.

[6] Labonte, R. and Laverack, G. (2001) Capacity building in health promotion, part 2; whose use? And with what measurement? Critecal Public Health, 11, 129-139. doi:10.1080/09581590110039847

[7] Laverack, G. (2001) An indentification and interpretation of the prganizational aspects of community empowerment. Community Development Journal, 36, 134-145. doi:10.1093/cdj/36.2.134

[8] Merzel, C. and Afflitti, J.D. (2003) Reconsidering community-based health promotion: Promise, performance, and Potential. American Journal of Public Health, 93, 557-574. doi:10.2105/AJPH.93.4.557

[9] Wandersman, A. and Florin, P. (2003) Community intervention and effective prevention. American Psychologist, 58, 441-448. doi:10.1037/0003-066X.58.6-7.441

[10] Labonte, R. and Laverack, G. (2001) Capacity building in health promotion, part 1; for whom? And for what purpose? Critecal Public Health, 11, 111-127. doi:10.1080/09581590110039838

[11] Toledo, M.E., et al. (2007) Achieving sustainability of community-based dengue control in Santiago de Cuba. Social Science \& Medicine, 64, 976-988. doi:10.1016/j.socscimed.2006.10.033

[12] Promprou, S., Jaroensutasinee, M. and Jaroensutasinee, K. (2005) Climatic factors affecting dengue heamorrhagic fever incidence in southern Thailand. Dengue Bulletin, 29, 41-48.

[13] Nguyen, M.-N., et al. (2005) Sustainability of the impact a public health intervention: Lessons learned from the Laval walking clubs experience. Health Promotion Practice, 6, 44-52. doi:10.1177/1524839903260144

[14] Bopp, M. and Bopp, J. (2002) Welcome to de Swamp: Measuring community capacity is fundamental to transformational work. The Four Worlds Centre for Development Learning, Canada.

[15] Labonte, R. and Laverack, G. (2001) Capacity building in health promotion, part 2; whose use? And with what measurement? Critical Public Health, 11, 129-139. doi:10.1080/09581590110039847

[16] Laverack, G. (2003) Building capable communities: Experiences in a rural Fijian context. Health Promotion International, 18, 99-106. doi:10.1093/heapro/18.2.99
[17] Laverack, G. and Wallerstein, N. (2001) Measuring community empowerment: A fresh look at organizational domains. Health Promotion International, 16, 179-185. doi:10.1093/heapro/16.2.179

[18] Suwanbamrung, C., et al. (2010) Community capacity for sustainable community-based dengue prevention and control: Study of a Sub-district in Southern Thailand. Asian Pacific Journal of Tropical Medicine, 3, 1-5.

[19] Suwanbamrung, C., et al. (2009) Application of a Dengue Community Capacity-Assessment Tool (DCCAT) for sustainable community-based dengue prevention and control. The 1st Annual International Graduate Research Conference on Social Sciences and Humanities Theme "Harmony in Diversity”, 2-3 April 2009. Bangkok.

[20] PAHO (2003) 44th Directing Council 55th Session of the Regional Committee. WHO, Washington DC.

[21] CDC (2003) Epidemic/Epizootic west Nile virus in the United States: Guidelines for surveillance, prevention, and control. Centers for Disease Control and Prevention, Colorado.

[22] Spark, R. (2003) Dengue fever management plan for North Queensland 2000-2005. Queensland Government, Queensland.

[23] Suwanbamrung, C., et al. (2008) The development of a tool to assess community capacity of sustainable community-based dengue prevention and control: A Study in Southern Thailand, in graduate school. Chulalongkorn University, Bangkok.

[24] Suwanbamrung, C., et al. (2011) A model of community capacity building for sustainable dengue problem solution in Southern Thailand. Health, 3, 584-601. doi:10.4236/health.2011.39100

[25] Suwanbamrung, C. (2010) Community capacity for sustainable community-based dengue prevention and control: Domain, assessment tool and capacity building model. Asia Pacific Journal Tropical Medicine, 3, 499-504. doi:10.1016/S1995-7645(10)60121-6

[26] Suwanbamrung, C., et al. (2009) Community capacity domains of dengue prevention and control. Asia Pacific Journal Tropical Medicine, 2, 50-57.

[27] Eisen, L., et al. (2009) Proactive vector control strategies and improved monitoring and evaluation practices for dengue prevention. Entomological Society of America, 46, 1245-1255.

[28] Focks, D.A. (2004) A review of entomological sampling methods and indicates for dengue vectors. WHO, Geneva.

[29] Norton, B.L., et al. (2002) Community capacity: Concept, theory, and methods. Emerging theory in health promotion practice and research. In: DiClemente, R., Crosby, R. and Kegler, M. Eds., Jossey-Bass Wiley, San Francisco. 